\title{
Shock propagation in a cemented tungsten carbide
}

\author{
G. J. Appleby-Thomas, ${ }^{1, a)}$ P. J. Hazell, ${ }^{1}$ C. Stennett, ${ }^{1}$ G. Cooper, ${ }^{1}$ K. Helaar, ${ }^{2}$ and \\ A. M. Diederen ${ }^{2}$ \\ ${ }^{1}$ Cranfield Defence and Security, Cranfield University, Shrivenham, Swindon SN6 8LA, United Kingdom \\ ${ }^{2}$ TNO Defensie en Veiligheid, Lange Kleiweg 137, P.O. Box 45, 2288 AA Rijswijk, The Netherlands
}

(Received 23 July 2008; accepted 22 January 2009; published online 24 March 2009)

\begin{abstract}
WC-based ceramic metal composites (cermets) are of great importance in both armor and munition design due to the combination of properties imparted by the presence of two different phases. WC-Co cermets are of interest in this area due to the hardness and strength imparted by the WC phase while the cementing Co matrix acts to increase plasticity and toughness. Here the dynamic response of G13 WC-Co manufactured by Kennametal Engineered Products B.V. was studied via a series of plate impact experiments involving both longitudinal and lateral gauges, which allowed determination of the $U_{s}-U_{p}$ relationship, measurement of a Hugoniot elastic limit of $3.3 \pm 0.2 \mathrm{GPa}$, measurement of a spall strength of $4.38 \mathrm{GPa}$, and an investigation of the stress dependence of shear strength in such a strong material. @ 2009 American Institute of Physics. [DOI: 10.1063/1.3087109]
\end{abstract}

\section{INTRODUCTION}

Cemented carbides-also known as hardmetals-are a group of refractory composites in which a hard carbide phase is held in situ or "cemented" by a binding phase, typically $\mathrm{Co}, \mathrm{Ni}$, or $\mathrm{Fe} .{ }^{1}$ In cemented carbides with a Co matrix, e.g., $\mathrm{WC}-\mathrm{Co}$, the hexagonal WC particles provide a high hardness and strength, while the Co matrix, which forms a continuous film around the WC particles, increases toughness and plasticity. ${ }^{1,2}$ The mechanical properties of WC-Co hardmetals are strongly dependent on both the WC particle/ crystallite size and the Co matrix content; for example, hardness and compressive strength both increase with either a reduction in Co content or increase in WC grain/crystallite size.

The high compressive strength of these materials, coupled with a relatively high density and good toughness, makes WC-Co hardmetals particularly attractive for ammunition applications. They are well known for defeating ceramic-faced armors where steel-cored projectiles would otherwise fail. ${ }^{3,4}$ Consequently, cemented carbides have been used since the Second World War in such projectiles as the $14.5 \mathrm{~mm} \times 114 \mathrm{~mm}$ BS41 armor-piercing (AP) round that was fielded by the Soviet army. In the past few years the use of tungsten carbide cores in the manufacture of AP bullets has become commonplace. This form of ammunition is rapidly replacing the previous generation of steel-cored AP bullets. For example, United States forces have adopted the M993 and M995 tungsten carbide-cored AP rounds into general use in the 7.62 and $5.56 \mathrm{~mm}$ calibers, respectively. Further, $12.7 \mathrm{~mm}$ caliber rounds with tungsten carbide cores are rapidly being adopted by European armies as a way of upgrading the effectiveness of AP bullets while using existing machine gun systems. Therefore there is a requirement to understand the dynamic behavior of such materials.

There has been a limited study of the dynamic behavior

\footnotetext{
a) Author to whom correspondence should be addressed. Tel.: +44 (0) 1793 784192. Electronic mail: g.applebythomas@cranfield.ac.uk.
}

of WC focused on ceramic (hot pressed) WC, ${ }^{5,6}$ with a more extensive body of work studying the shock response of WC-Co cermets. ${ }^{7-10}$ Data on WC-Co with Co contents of 5-6 wt \% covering particle velocities up to $1820 \mathrm{~m} / \mathrm{s}$ have been presented and have shown typical $U_{s}-U_{p}$ and $P$ $-V / V_{0}$ relationships within this regime. ${ }^{7,8}$ Additionally Grady $^{7}$ studied the response of a 3-4 wt \% Ni matrix-based WC cermet extracted from a $14.5 \mathrm{~mm}$ AP (BS-41) bullet core. The Co-based cermet exhibited a Hugoniot elastic limit (HEL) of around 4.1 GPa with the Ni-based cermet showing broadly similar behavior, although no precise HEL was found. $^{7}$ The Ni-based cermet showed a spall strength of 3.6 $\mathrm{GPa}$, while that with a Co matrix showed a lower spall strength of $2.8 \mathrm{GPa}$. An unusual three-stage shock rise was noted within the cermet in all cases: ${ }^{7}$ (1) the elastic rise to the HEL, (2) a plastic "ramp" in stress after the HEL, and (3) a plastic rise to the Hugoniot stress. This unusual plastic ramp was attributed to deformation hardening, although other potential causes including pressure hardening and void generation were considered. Similar behavior has also been observed in WC ceramics, ${ }^{5,6}$ although here an alternate explanation for the post-HEL ramp in the form of collapse of pores within the WC (at greater shock velocities the ramp timescales decreased) was proposed. ${ }^{6}$

No lateral gauge studies on WC-Co were found in the literature; however, Millett et $a .^{6}{ }^{6}$ conducted plate impact experiments on a WC ceramic to investigate lateral stress and shear strength. A decrease in shear strength behind the main shock (corresponding to an increase in lateral stress) was observed and attributed to plasticity due to dislocation movement and generation.

In this study a WC-Co cermet grade G13 was investigated over the velocity regime $199-857 \mathrm{~m} / \mathrm{s}$. A grain size distribution of 2-4 $\mu \mathrm{m}$ was quoted for the G13 WC-Co, which was supplied in the form of ready polished disks of up to $65 \mathrm{~mm}$ diameter with a planarity of $<5 \mu \mathrm{m}$. The supplied G13 had a slightly higher Co composition than those considered in the literature ${ }^{7,8}$ at $8.5 \mathrm{wt} \%$. The $U_{s}-U_{p}$ relationship is determined based on strain gauge data from a series of 
TABLE I. Elastic properties of G13 WC-Co.

\begin{tabular}{lccccc}
\hline \hline Material & $\begin{array}{c}\rho_{0} \\
\left(\mathrm{~g} / \mathrm{cm}^{3}\right)\end{array}$ & $\begin{array}{c}c_{L} \\
(\mathrm{~mm} / \mu \mathrm{s})\end{array}$ & $\begin{array}{c}c_{S} \\
(\mathrm{~mm} / \mu \mathrm{s})\end{array}$ & $\begin{array}{c}c_{B} \\
(\mathrm{~mm} / \mu \mathrm{s})\end{array}$ & $\nu$ \\
\hline G13 WC-Co & 14.74 & 6.83 & 4.09 & 4.94 & 0.22 \\
\hline \hline
\end{tabular}

plate impact experiments and lateral stress/shear strength evolution investigated using lateral gauges. Additionally estimates of both the WC-Co HEL and spall strength are made.

\section{EXPERIMENTAL}

Longitudinal and shear wave velocities were measured ultrasonically using a Panametrics 5077PR pulse receiver in the pulse-echo configuration and density was measured using a Micrometrics AccuPyc 1330 gas pycnometer. Measured and subsequently calculated elastic properties of G13 WC-Co are shown in Table I.

Scanning electron micrographs of perpendicular sections through supplied G13 WC-Co are shown in Fig. 1 and show a typical hardmetal two-phase structure with no notable anisotropy and a slightly larger than expected bimodal WC crystallite/grain size distribution of $<1-7.5 \mu \mathrm{m}$.

Equation of state data were determined experimentally using plate impact experiments ${ }^{11,13,14}$ conducted on a $5 \mathrm{~m}$ barrel $50 \mathrm{~mm}$ diameter gas gun. ${ }^{12} \mathrm{WC}-\mathrm{Co}$ disks were mounted together with a cover plate and a $12 \mathrm{~mm}$ thick backing polymethylmethacrylate (PMMA) block with two Vishay Micro-Measurements type LM-SS-125CH-048 Manganin strain gauges in between as shown in Fig. 2. The cover plate was included in order to provide protection to the front surface gauge, with the material chosen to be that of the flyer in line with the impedance matching technique. ${ }^{8,14}$
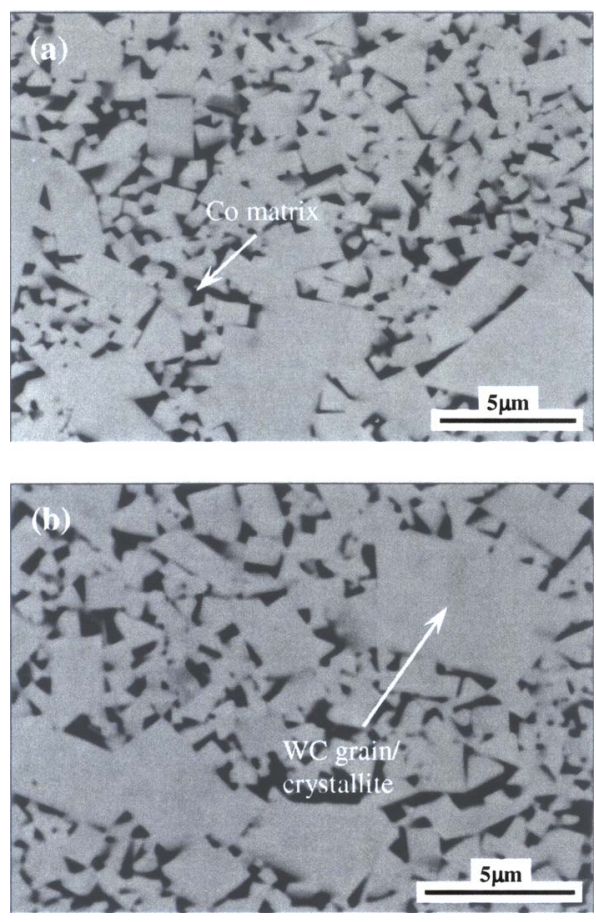

FIG. 1. (Color online) Backscattered scanning electron images of throughthickness (a) and top surface (b) sections of a sample G13 WC-Co target.

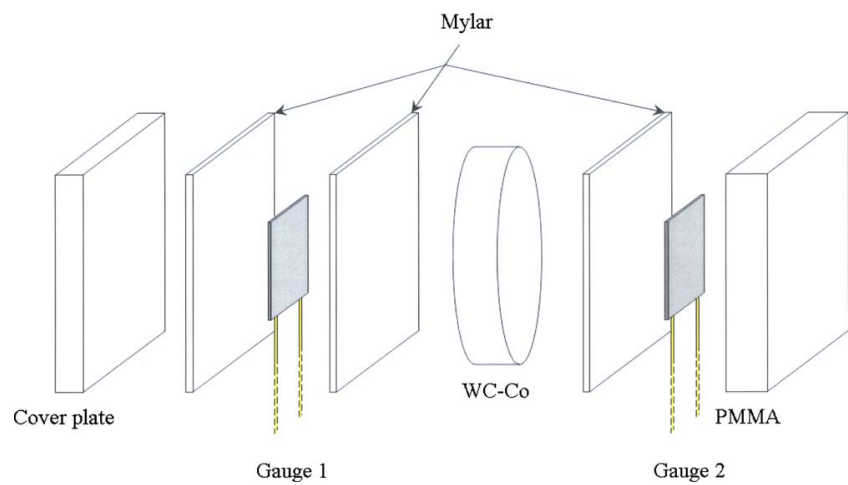

FIG. 2. (Color online) Schematic illustration (exploded) of target package.

Mylar thicknesses of 25-100 $\mu \mathrm{m}$ were employed to insulate/protect the Manganin gauges, and an epoxy adhesive (Loctite $^{\text {TM }} 0151$ Hysol Epoxi-Patch) with a similar shock impedance to both the Mylar and the gauge assembly itself was chosen for package assembly. The total separation of the front and rear surface gauges was determined by measuring the target thickness accurately before the experiment using a micrometer and by making allowance for the thickness of Mylar encapsulation in front/behind the gauges as appropriate. Wave velocities were then calculated from transit times for the wave, which were measured based on initial wave arrival at the front and back surface gauges successively. In addition, maximum/minimum wave velocities about the measurement point for both gauges were determined and used in subsequent error calculations. At higher impact stresses several of the observed wave traces exhibited ramps/ changes in gradient during the initial rise to a peak stress. In such cases the measured wave velocity therefore varied depending on the position at which wave arrival was measured at the two gauges. Where such a change in gradient was apparent, wave arrival was measured between corresponding points on the two traces at the measured stress corresponding to the first change in gradient on either gauge.

Data recording using a $1 \mathrm{GHz}$ oscilloscope was initiated by shorting of a pair of trigger pins located in a target ring used for target package mounting-the entire arrangement was subsequently fixed onto a barrel extension. All surfaces in contact with the target package or barrel were finished to a flat surface with a planarity of $\leq 5 \mu \mathrm{m}$. Gun alignment shots comprising a target of three ionization pins spaced across a $\mathrm{Cu}$ disk undertaken during the course of this investigation confirmed that these measures resulted in a misalignment consistently $<1.5 \mathrm{mrad}$ s. A series of graphite pins was used to calculate the terminal velocity of the projectile. The target assembly, excluding the barrel extension, is shown schematically in Fig. 3.

Balancing and calibration of the gauges were undertaken following Rosenberg et al., ${ }^{13}$ which provided a ready approach to scale measured changes in voltage with time recorded during the plate impact experiments to changes in stress within the Manganin gauges; and subsequent data interpretation following a slightly modified version of the impedance matching technique. ${ }^{8,14,15}$ Essentially, the intercept between an inverted form of the known polynomial of the particle velocity-pressure $\left(U_{P}-P\right)$ Hugoniot of the flyer ma- 


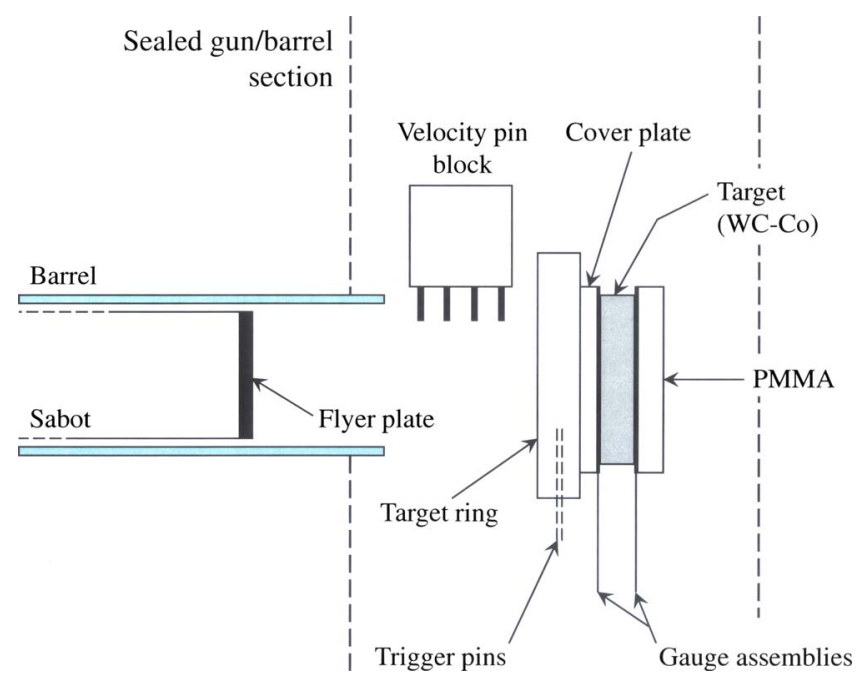

FIG. 3. (Color online) Experimental setup for the plate impact studies.

terial was equated to the Rayleigh line of the target material - in both cases with the data offset by the impact velocity of the flyer plate, leading to the relationship shown in Eq. (1). This was possible because, by definition, the particle velocity within the flyer, cover, and target materials was equivalent.

$$
\rho_{0} U_{S}\left(U_{P}^{\prime}-V_{\text {impact }}\right)=A U_{P}^{\prime 2}-B U_{P}^{\prime}+C,
$$

where $\rho_{0}$ is the density of the WC-Co target, $U_{S}$ is the measured shock velocity in the $\mathrm{WC}-\mathrm{Co}$ target, $U_{P}^{\prime}$ is the effective particle velocity, $V_{\text {impact }}$ is the impact velocity of the projectile, and $A, B$, and $C$ are the (known) polynomial constants of the $U_{P}-P$ Hugoniot for the flyer material.

Equation (1) was then rewritten as a polynomial in $U_{P}^{\prime}$ and solved using a least-squares-fitting approach. Subsequently, the calculated $U_{P}^{\prime}$ value was converted to the actual particle velocity in the WC-Co target $\left(U_{P}\right)$ by addition of the flyer impact velocity according to

$$
U_{P}=U_{P}^{\prime}+V_{\text {impact }}
$$

Lateral gauge shots employed a modified WC-Co target sectioned and ground to a flatness of $\leq 5 \mu \mathrm{m}$ perpendicular to the main WC-Co impact faces with a Micro-Measurements type J2M-SS-580SF-025 lateral gauge embedded $5 \mathrm{~mm}$ from the impact face encapsulated in $25 \mu \mathrm{m}$ thick Mylar. This arrangement is shown schematically in Fig. 4. Lateral traces were interpreted using a modified form of the impedance matching technique, developed by Rosenberg and Partom, ${ }^{16}$ which was based on the assumption that the strain in a thin (foil) laterally embedded gauge was equivalent to that of the surrounding material. This approach was adapted to take account of both the elastic-plastic response of the Manganin gauge used $^{17}$ as well as the pressure dependence of its response at stresses below its elastic limit. ${ }^{18}$ The configuration shown in Fig. 4 was typically assembled with a rear surface longitudinal gauge insulated by a $25 \mu \mathrm{m}$ thick Mylar and retained in situ by a PMMA backing block. This second gauge provided time of arrival data for the shock enabling further $U_{s}-U_{p}$ data to be established in the course of the lateral gauge shots according to the impedance matching

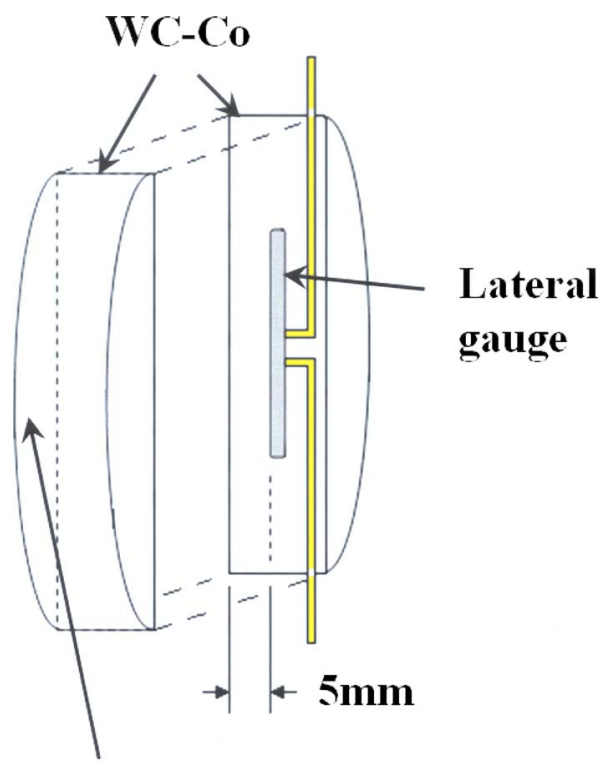

\section{Impact face (hidden)}

FIG. 4. (Color online) Schematic showing location of lateral gauge within the WC-Co target.

technique for longitudinal gauges set out previously. It should also be noted that while for the longitudinal shots cover plates of the same material as the flyer were selected in order to provide protection for the gauge, for the lateral shots no cover plate was necessary as the gauges were positioned sufficiently far from the impact surface $(5 \mathrm{~mm})$ to survive the initial impact. Consequently, for the lateral shots, the cover material was treated for impedance matching purposes as WC-Co.

Flyer velocities in the range 199-857 m/s were investigated, inducing longitudinal stresses of 2.5-23.5 GPa in the WC-Co. Concurrently, lateral stress evolution was investigated for impact velocities of 199-656 m/s.

\section{RESULTS AND DISCUSSION}

A total of 15 shots, 8 using longitudinal gauges and 7 using either lateral gauges alone or combinations of lateral/ longitudinal gauges, were undertaken. A summary of the experimental conditions and key results from these tests is presented in Table II. $U_{S}-U_{P}$ data were extracted from both the longitudinal shots and, in three cases, from targets incorporating a lateral gauge to which a rear surface longitudinal gauge with PMMA backing was attached in order to allow calculation of the transit time for the wave. In one case, for an impact velocity of $453 \mathrm{~m} / \mathrm{s}$, uncertainty in the position of the front lateral gauge following target assembly led to indeterminate $U_{S}-U_{P}$ data and consequently such data are not included either in Table II or the subsequently derived Hugoniot. Additionally, in two cases for impact velocities of 541 and $646 \mathrm{~m} / \mathrm{s}$, rear surface gauge failure led to no $U_{S}-U_{P}$ or $\sigma_{X}$ data being determined and such data are therefore not included in Table II. One other lateral shot was also undertaken with no rear surface gauge. In this case no $U_{S}-U_{P}$ or $\sigma_{X}$ data were directly measured, and consequently these data are not included for this shot in Table II. Instead, where $\sigma_{x}$ 
TABLE II. Summary of experimental results.

\begin{tabular}{|c|c|c|c|c|c|c|}
\hline $\begin{array}{l}V_{\text {impact }} \\
(\mathrm{m} / \mathrm{s})\end{array}$ & $\begin{array}{c}\text { Flyer } \\
\text { material }\end{array}$ & $\begin{array}{l}\text { Flyer thickness } \\
\qquad(\mathrm{mm})\end{array}$ & Type of shot & $\begin{array}{c}U_{P} \\
(\mathrm{~mm} / \mu \mathrm{s})\end{array}$ & $\begin{array}{c}U_{S} \\
(\mathrm{~mm} / \mu \mathrm{s})\end{array}$ & $\begin{array}{c}\sigma_{X} \\
(\mathrm{GPa})\end{array}$ \\
\hline 199 & Dural & 10 & Lateral/longitudinal & 0.025 & 6.87 & 3.81 \\
\hline 201 & Dural & 5 & Longitudinal & 0.026 & 6.70 & 2.43 \\
\hline 352 & Dural & 10 & Latera1/longitudinal & 0.046 & 6.87 & 4.79 \\
\hline 407 & $\mathrm{Cu}$ & 10 & Longitudinal & 0.118 & 6.48 & 10.34 \\
\hline 442 & Dural & 10 & Lateral/longitudinal & 0.059 & 6.79 & 6.70 \\
\hline 453 & $\mathrm{Cu}$ & 10 & Lateral/longitudinal & Uncertain gauge spacing & Uncertain gauge spacing & 14.55 \\
\hline 455 & Dural & 10 & Lateral & $\mathrm{n} / \mathrm{a}$ & $\mathrm{n} / \mathrm{a}$ & $\mathrm{n} / \mathrm{a}$ \\
\hline 484 & $\mathrm{Cu}$ & 5 & Longitudinal & 0.139 & 6.66 & 12.89 \\
\hline 492 & Dural & 10 & Longitudinal & 0.068 & 6.76 & 6.91 \\
\hline 541 & $\mathrm{Cu}$ & 5 & Lateral/longitudinal & Gauge failure & Gauge failure & Gauge failure \\
\hline 644 & $\mathrm{Cu}$ & 5 & Longitudinal & 0.193 & 6.49 & 23.18 \\
\hline 656 & $\mathrm{Cu}$ & 10 & Lateral/longitudinal & Gauge failure & Gauge failure & Gauge failure \\
\hline 714 & $\mathrm{Cu}$ & 5 & Longitudinal & 0.224 & 6.15 & 22.61 \\
\hline 849 & $\mathrm{Cu}$ & 5 & Longitudinal & 0.279 & 5.93 & Gauge failure \\
\hline 857 & $\mathrm{Cu}$ & 5 & Longitudinal & 0.283 & 5.87 & 26.32 \\
\hline
\end{tabular}

data for strength calculations were subsequently required these were derived from the measured WC-Co Hugoniot based on the measured flyer impact velocity.

A typical front gauge trace for a longitudinal experiment with an impact velocity of $484 \mathrm{~m} / \mathrm{s}$ is shown in Fig. 5. The initial shock rise time was relatively slow at $\sim 140 \mathrm{~ns}$, attributed to the thick Mylar protection experimentally found to be necessary to prevent premature gauge failure. A constant maximum stress was subsequently obtained before releases from the rear of the flyer led to gauge failure. Just before the shock began to ramp up a dip below zero stress was observed at (a). This has been seen elsewhere and has been attributed to an increase in capacitance between the gauge and cover plate as the cover plate is accelerated toward the gauge. ${ }^{19}$ Noise at (b) and (c) just before the maximum stress plateau appeared electrical in nature and was attributed to a capacitance effect between the cover plate and insulated gauge caused by ionization of the propellant gas around the incident flyer plate. Additionally the release appeared two-stage in nature with a presumed high-stress elastic region above (d) and an elastic-plastic region below. However, no attempt was made to extract WC-Co specific information from this region as it was noted that the apparent elastic-plastic release

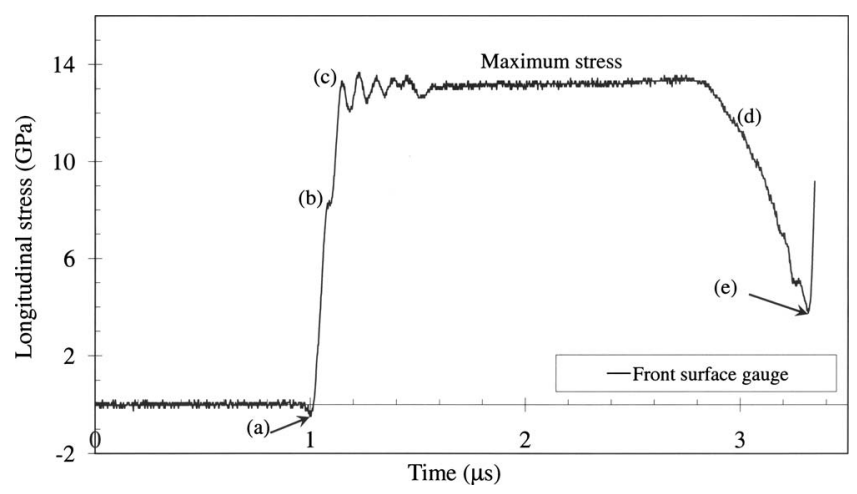

FIG. 5. Front surface trace, $5 \mathrm{~mm} \mathrm{Cu}$ flyer, $V_{\text {impact }}=484 \mathrm{~m} / \mathrm{s}$, and $6.32 \mathrm{~mm}$ thick WC-Co target. at (d) may also be linked to the Manganin gauge response as well as that of the WC-Co target. Finally, gauge failure is observed to occur at (e).

For comparison, stress histories from both the front and back surface gauges for a higher velocity, $V_{\text {impact }}=644 \mathrm{~m} / \mathrm{s}$, shot are presented in Fig. 6. An arrow has been included in Fig. 6 showing the separation, $\Delta t_{\text {shock }}$, between the two traces used to calculate the wave velocity (based on the measured target thickness, as discussed previously) and, therefore, via the impedance matching technique, the particle velocity in the WC-Co. Premature front gauge failure occurred due to substrate conduction as the stress approached $20 \mathrm{GPa}$. Here the back surface stresses have been scaled to represent approximately those in the $\mathrm{WC}-\mathrm{Co}$ target using

$$
\sigma_{\mathrm{WC}-\mathrm{Co}}=\frac{1}{2} \frac{\left(Z_{\mathrm{WC}-\mathrm{Co}}+Z_{\mathrm{PMMA}}\right)}{Z_{\mathrm{PMMA}}} \sigma_{\mathrm{PMMA}},
$$

where $\sigma_{\mathrm{WC}-\mathrm{Co}}$ is the stress in the $\mathrm{WC}-\mathrm{Co}, \sigma_{\mathrm{PMMA}}$ is the stress in the PMMA, $Z_{\mathrm{WC}-\mathrm{Co}}$ is the impedance of the $\mathrm{WC}-\mathrm{Co}$, and $Z_{\mathrm{PMMA}}$ is the impedance of the PMMA. In general, $Z=\rho_{0} U_{s}$, where $\rho_{0}$ is the material density and $U_{s}$ is the wave velocity measured at the position shown in Fig. 6.

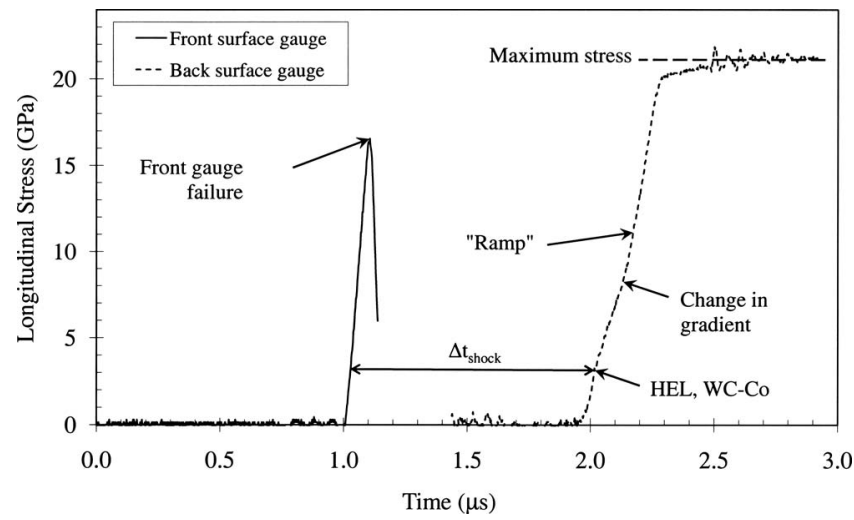

FIG. 6. Front and rear surface gauge traces (stress in WC-Co), $5 \mathrm{~mm} \mathrm{Cu}$ flyer, $V_{\text {impact }}=644 \mathrm{~m} / \mathrm{s}$, and $6.33 \mathrm{~mm}$ thick WC-Co target. 


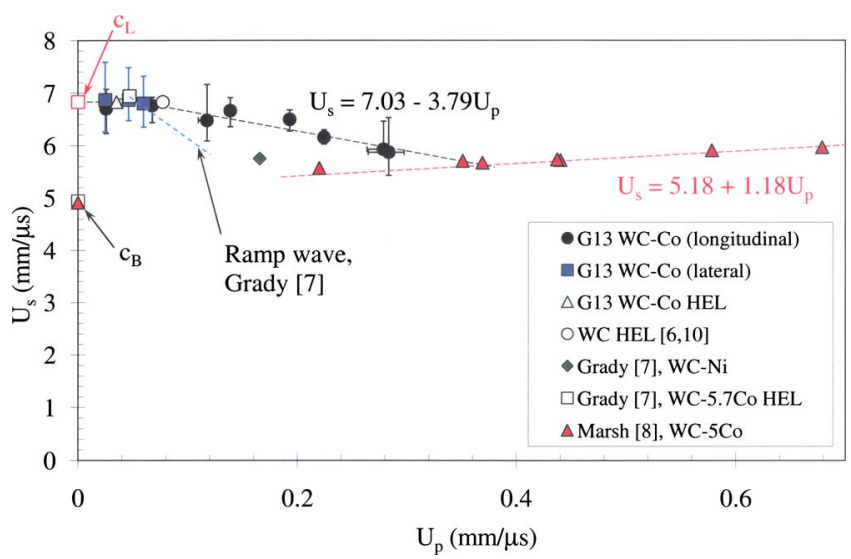

FIG. 7. (Color online) $U_{s}-U_{p}$ relationship for G13 WC-Co plus comparative results (Refs. 6-8 and 10).

The use of Eq. 3 in Fig. 6 to calculate the maximum stress experienced by the WC-Co from the lower PMMA stress confirmed that the peak stress exceeded the Manganin strain gauge upper limit of $\sim 20 \mathrm{GPa}$ explaining the front gauge failure. The rear surface trace exhibits three distinct regions during the initial rise to the maximum stress each separated by a change in gradient. These comprised (1) an initial purely elastic linear ramp up to the HEL of WC-Co, (2) an elastic-plastic response up to a stress $\sim 8 \mathrm{GPa}$ where the gradient was observed to change, and (3) a "ramped" plastic region up to the maximum recorded stress. The final plastic ramp up to a full shock apparent for similar grades of WC-Co elsewhere ${ }^{7}$ was not present on any of the recorded traces. As shown later in this paper this was because all tests undertaken lie on either the purely elastic or the plastic ramp regimes. Similar ramped regions immediately following the HEL were observed by $\mathrm{Grady}^{7}$ and attributed to elasticplastic hardening behavior arising from deformation hardening (compression of the WC particles). Figure 6 gave a WC-Co HEL of $\sim 3.2 \mathrm{GPa}$ and overall an average WC-Co HEL of $3.3 \pm 0.2 \mathrm{GPa}$ was determined. This is lower then the HEL determined by $\mathrm{Grady}^{7}$ for a similar WC-Co grade. This difference was attributed to the lower strength of the G13 WC-Co, a product of its higher Co content $(8.5 \%$ as opposed to $5.7 \%$ ). The change in gradient just above the WC-Co HEL in Fig. 6 is found to occur at $\sim 8 \mathrm{GPa}$. This is approximately equal to the HEL of WC ceramic. ${ }^{10}$ It is proposed that the presence of two different points of inflection corresponding to the HEL values of both $\mathrm{WC}-\mathrm{Co}$ and $\mathrm{WC}$ may be attributed to dispersion of the elastic wave within the cermet as the WC particles were compressed. Essentially here the region between the HEL of WC-Co and $\mathrm{WC}$ is interpreted as a diffuse elastic limit, with the cermet reaching its elastic limit first, but with the WC particles remaining elastic up to $\sim 8 \mathrm{GPa}$.

The calculated $U_{s}-U_{p}$ relationship is presented in Fig. 7 along with additional data from the literature as appropriate. The error bars are based on the assessed accuracy of the measured wave velocities. As previously discussed, the measured wave velocities depended on the position at which $\Delta t_{\text {shock }}$ (shown in Fig. 6) was assessed. Maximum/minimum likely wave velocities were measured by altering the position at which wave arrival was assumed to occur on each gauge. From Eq. (1) it was observed that the calculated particle velocities depended on the measured wave velocities. Consequently, in each case, the assessed range of possible wave velocities was used to calculate maximum/minimum values for the particle velocity. These calculations are reflected in the error bars included on the experimental data in Fig. 7.

The $U_{s}-U_{p}$ relationship in Fig. 7 is divided into three sections, an initial constant $U_{s}$ region, which appears entirely elastic up to $U_{p}=0.03 \mathrm{~mm} / \mu \mathrm{s}$, and then two sections above/ below $U_{p}=0.37 \mathrm{~mm} / \mu \mathrm{s}$, with positive and negative gradients, respectively. In the two sloped regions the best fit takes the general form shown in Eq. (4), with the intercepts approximating the measured sound velocities.

$$
U_{s}=c_{0}+S U_{p},
$$

where, in general, $c_{0}$ is the target material sound speed and $S$ is a shock parameter.

Further, the particle velocity at the HEL of both WC-Co and $\mathrm{WC}$ was calculated using ${ }^{14}$

$$
U_{p}=\frac{\sigma_{\mathrm{HEL}}}{\sqrt{\rho E}},
$$

where $E$ is Young's modulus (600.1 GPa for G13 WC-Co).

The observed trilinear $U_{s}-U_{p}$ relationship comprises an initial elastic response up to $U_{p}=0.03 \mathrm{~mm} / \mu \mathrm{s}$, followed by a ramped region, which intercepts the principal WC-Co Hugoniot determined by Marsh $^{8}$ between the two sloped lines of best fit in Fig. $7\left(U_{p}=0.37 \mathrm{~mm} / \mu \mathrm{s}\right.$ and $U_{s}$ $=5.62 \mathrm{~mm} / \mu \mathrm{s})$. None of the experimental datapoints from this work lie on the principal Hugoniot, instead all lie within either the initial elastic region or the subsequent (plastic) ramped region. This explains the lack of a final plastic rise to a shock in any recorded trace (for example, Fig. 6). Given a WC-Co HEL of $3.3 \mathrm{GPa}$, the maximum particle velocity at which G13 WC-Co remains elastic was estimated from Eq. (5) as $0.035 \mathrm{~mm} / \mu \mathrm{s}$ (as the wave is still elastic up to the HEL). This is plotted in Fig. 7 and is in good agreement with the intercept between the elastic and plastic regions (e.g., $\left.U_{p}=0.03 \mathrm{~mm} / \mu \mathrm{s}\right)$.

However, observation of the experimental data in Fig. 7 showed that a good fit through the plastic data could also be achieved if an intercept with the purely elastic regime of $U_{p}=0.08 \mathrm{~mm} / \mu \mathrm{s}$ was assumed. Using literature data for WC, ${ }^{6,10}$ combined with Eq. (5), the particle velocity at the HEL of pure WC was calculated as $0.08 \mathrm{~mm} / \mu$ s (plotted in Fig. 7). This ties in well with the observed diffuse elastic limit running between the HEL values of WC-Co and WC apparent in the rear surface trace in Fig. 6. This gives credence to the suggestion that rather than a precise elastic limit, the presence of the WC particles leads to an effective dispersion of the elastic wave, with the WC element of the cermet continuing to behave elastically beyond the elastic limit of the cermet. This link between the elastic response and the composite nature of the WC-Co cermet has been observed elsewhere, e.g., by Klein et $a l .{ }^{20}$ for TiC-steel systems.

While trilinear responses are not readily apparent in the literature, the bilinear response seen in Fig. 7 above $U_{p}$ 
$=0.03 \mathrm{~mm} / \mu \mathrm{s}$ has been seen elsewhere. Grady ${ }^{7}$ suggested the existence of a ramp wave between the Hugoniot for WC-Co and the calculated HEL, as illustrated schematically in Fig. 7. However, Grady ${ }^{7}$ was only able to obtain a small number of datapoints and only one (for WC-Ni) showed significant deviation from the main Hugoniot. Therefore the precise nature of this ramp wave is open to interpretation. Two different mechanisms have been proposed in the literature to explain bilinear behavior: (a) the completion of a shock induced phase change with the change initially lowering the bulk sound speed, ${ }^{21}$ and (b) the stress dependence of the elastic precursor velocity leading to its measurement instead of the main shock for $\sigma<\sigma_{\mathrm{HEL}}$ (Ref. 22) (TiB2). Additionally, Zhang et $_{\text {al. }}{ }^{22}$ suggested that a two-stage Hugoniot is indicative of retention of material shear strength in the plastic region. If a deformation hardening effect (compression of the WC particles) occurred, which reached saturation at $U_{p}=0.37 \mathrm{~mm} / \mu \mathrm{s}$, then some credence might be given to mechanism (a). It proved difficult to discern the HEL on rear surface traces at impact velocities $<450 \mathrm{~m} / \mathrm{s}$ even where front surface gauge failure indicated $\sigma \gg \sigma_{\mathrm{HEL}}$. It is therefore possible that at least part of the ramp from the HEL of WC-Co back to the principal Hugoniot in Fig. 7 may be attributed to measurement of the elastic precursor. Additionally, the apparent dispersion of the elastic wave alluded to in Figs. 6 and 7 implies that an elastic precursor would still have been dominant up to at least $U_{p}=0.08 \mathrm{~mm} / \mu$ s (corresponding to the HEL of pure WC). ${ }^{6}$ Po,14 However, at higher velocities, once both the G13 WC-Co and pure WC HELs become apparent the continued bilinear nature of the $U_{s}$ $-U_{p}$ relationship above the HEL of WC cannot be fully explained. Klein et $a l .{ }^{20}$ found that the dynamic response of the TiC-steel system was largely dependent on the interaction between the two constituent phases. At impact stresses above the HEL shock compression resulted in the TiC ceramic being entirely crushed (a phenomenon also alluded to by Meyers ${ }^{14}$ ), reducing the effective cross-sectional area of the targets to that of the metal matrix. In the case of WC-Co, inverse behavior might be expected as failure has been shown to occur in the Co matrix meaning that the response of the (effectively sintered) WC particles is likely to dominate at higher impact velocities. This idea of a dynamic response arising from the interplay of the two phases, with the response of the WC particles becoming dominant at higher impact velocities, appears plausible. It ties in well with the observed dispersion of the elastic limit in Figs. 6 and 7 between those of $\mathrm{WC}-\mathrm{Co}$ and pure $\mathrm{WC}$ and represents another possible explanation for the observed nonelastic bilinear behavior.

A distinct unloading/reloading signal was apparent on the rear surface gauge of the $857 \mathrm{~m} / \mathrm{s}$ shot as shown in Fig. 8 . This represented a differential of $4.38 \mathrm{GPa}$, a value in good agreement with the measured spall strength values of 2.8-3.5 $\mathrm{GPa}$ for similar grades of $\mathrm{WC}-\mathrm{Co}$, namely, $\mathrm{WC}-\mathrm{Ni}$ and WC-Co recorded elsewhere. ${ }^{7}$ Therefore, given the high particle/shock velocity, the relatively high thickness of the target (10.36 mm as compared to $\sim 6.2 \mathrm{~mm}$ in most cases), and the good agreement with literature data, this signal was assumed to represent dynamic spall of the G13 WC-Co. ${ }^{7,21}$

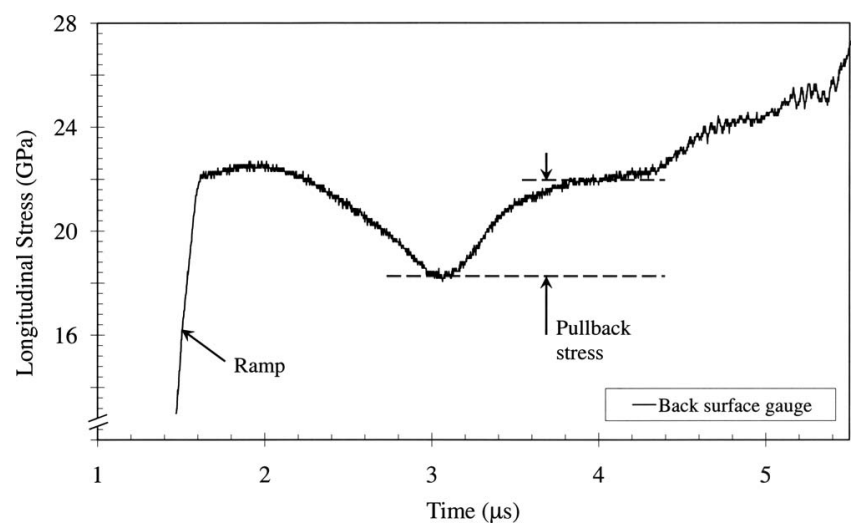

FIG. 8. Rear surface trace, $5 \mathrm{~mm} \mathrm{Cu}$ flyer, $V_{\text {impact }}=857 \mathrm{~m} / \mathrm{s}$, and $10.36 \mathrm{~mm}$ thick WC-Co target (ramped region attributed to arrival of the elastic precursor).

Lateral gauge traces for the five lateral experiments undertaken are shown in Fig. 9. The relatively slow rise times of $>300 \mathrm{~ns}$ are typical and have been observed elsewhere. ${ }^{18}$ A ramp/change in gradient is apparent in all traces during the shock arrival. This is a feature observed in similar results for monolithic WC, ${ }^{6}$ where it was attributed to the collapse of surface pores during shock arrival. Millett et al. ${ }^{6}$ also proposed that the rate at which this hardening behavior (e.g., pore collapse) occurs should increase with shock velocity. They linked this phenomenon to observed decreases in the duration and accompanying increases in gradient of the initial ramp in monolithic WC. The gradient of the ramp observed at the beginning of the traces shown in Fig. 9 is also seen to increase with impact velocity while the duration of the ramp is seen to simultaneously decrease. Given the observations by Millett et al. ${ }^{6}$ it therefore seems reasonable to conclude, based on the behavior of the ramp observed at the beginning of the lateral gauge traces, that some hardening behavior is occurring with increased impact stress. Unlike the longitudinal case in Fig. 5, this is followed by two different effects: (1) at lower impact velocities a reduction in stress, and (2) at higher velocities a steady increase in stress; in both cases this continues until either gauge failure when $\sigma_{x}>\sim 20 \mathrm{GPa}$ or a release ends the pulse.

Maximum shear strength, $\tau$, is related to the longitudinal stress $\left(\sigma_{x}\right)$ and the transverse stress $\left(\sigma_{y}\right)$ by

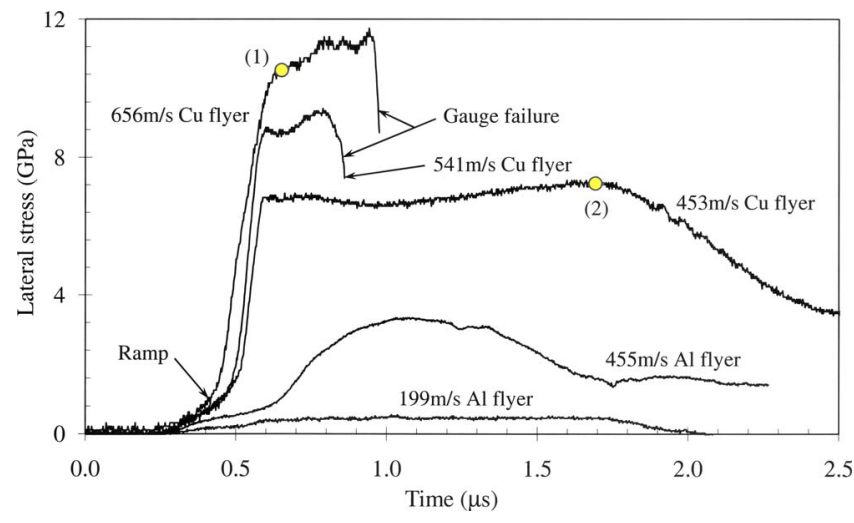

FIG. 9. (Color online) Lateral gauge traces for various impact velocities. 


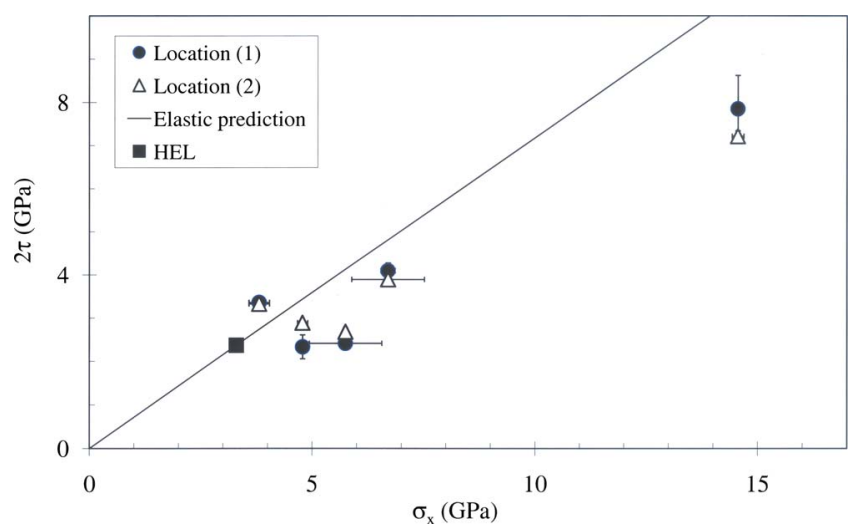

FIG. 10. (Color online) Variation of shear strength with impact stress for G13 WC-Co at locations (1) and (2) in Fig. 9.

$$
\tau=\frac{\sigma_{x}-\sigma_{y}}{2}
$$

Inertial confinement means that the longitudinal gauges, while responding to a change in pressure in the Manganin, are only measuring a one-dimensional (1D) responsenamely, the true longitudinal stress. This has been shown in Figs. 5 and 6 to remain constant for the duration of the shock (e.g., once the Hugoniot stress is reached). Consequently, from Eq. (6), the steady increase in magnitude of $\sigma_{y}$ behind the shock in Fig. 9 visible once the shock had ramped up implied a reduction in shear strength. Following the arrival of the main shock front maximum shear strength values at locations (1) and (2) for each trace in Fig. 9 (indicated by circles representing minimum/maximum lateral stresses, respectively, with data included for point (2) subject to gauge survival) were calculated and are presented in Fig. 10. A predicted isotropic elastic WC-Co response based on Eq. (6), which depends on Poisson's ratio $\nu$, is also included for the purpose of comparison. ${ }^{6,16}$ Additionally, the measured HEL with the associated shear strength calculated using the following equation is also included:

$$
2 \tau=\frac{1-2 \nu}{1-\nu} \sigma_{x} .
$$

This plot shows an increase in shear strength with impact stress. The movement of the datapoints from locations (1) and (2) below the elastic prediction with increasing stress indicates the onset of plastic deformation. Therefore, the intersection between the elastic prediction and the measured shear strength data should be the point of departure from the elastic curve, namely, the HEL. While no data from locations (1) or (2) are lower than the HEL, it is apparent that best-fit lines through these data in Fig. 9 (neither line is included for clarity) would lie either at or just below the HEL. E.g., if the HEL is included as a datapoint, the intercepts with the elastic prediction for the data from both locations (1) and (2) would be at $\sigma_{x}=2.48$ and $3.41 \mathrm{GPa}$, respectively. Nonetheless, despite the fact that the intercept from point (1) lies outside the error margin of the measured HEL, the data from both locations that sit on the elastic prediction confirm the order of magnitude of G13's HEL, thereby increasing confidence in the measured value. Additionally, slight evidence of the re- duction in shear strength behind the shock previously described is highlighted by the tendency of the points sampled at location (2) in Fig. 9 to stray below those from location (1) with increasing impact stress. However, insufficient data at higher stresses exist to quantify this effect.

Similar results for monolithic WC (Ref. 6) were attributed to plasticity in the target material as the shock wave progressed increasing lateral stress and therefore acting to reduce shear strength. In particular, plastic deformation was favored over an alternate explanation of the coalescence of cracks formed at the front of the shock leading to a reduction in shear strength [and therefore from Eq. (6) an increase in lateral stress] behind due to the presence of such weakening phenomenon below the HEL of WC. This was accounted for by the realization that the HEL represents an average stress across all crystallographic directions meaning that in the complex ordered hep structure of WC yielding of a proportion of grains orientated favorably in one direction below the HEL would be possible. This argument was used to justify the suggestion that the observed reduction in shear strength behind the shock both in this region and above the HEL was attributable to plastic deformation. ${ }^{6}$ While the lateral gauge traces shown in Fig. 9 have a similar form to those reported elsewhere, ${ }^{6,15}$ recent work by Winter and Harris ${ }^{23}$ has suggested that a modification of both the explanation for the observed reduction in shear strength and the interpretation of lateral gauge results is required. Simulations of plate impact experiments were used to investigate the propagation of shocks through both a so-called matrix material and a matrix material with an embedded fluid layer (analogous to an encapsulated gauge). These showed that the presence of a fluid layer leads to significant modifications of the nature of the shock front, with the shock in the fluid layer moving at a different velocity to the surrounding matrix. When the shock moves faster in the fluid layer a continual rise in lateral gauge stress until release results, while when this shock lags the matrix shock front an initial ramp to a peak followed by a steady decrease in magnitude to release is observed. The results of these models are consistent with the experimental data in Fig. 9 if it is assumed that the shock moves more slowly through the encapsulated gauge layer than the surrounding $\mathrm{WC}-\mathrm{Co}$ at lower stresses and faster at higher stresses. Such stress dependence was indeed noted by Winter and Harris for $\mathrm{Ta}^{23}$ Recent work by Winter et al. $^{24}$ has attempted to confirm this result by comparison to experiments that involved measurements independent of lateral gauge responses. The AWE heterodyne velocimetry (Het-V) technique was employed to investigate the recession velocity (which is directly proportional to particle velocity) at two different points, one central to the impact axis and one further out, on the rear surface of both a Ta and a steel target following impact with $\mathrm{Cu}$ flyers at $\sim 300 \mathrm{~m} / \mathrm{s}$. In each case, the target was assembled into two halves joined together by an intermediate $100 \mu \mathrm{m}$ thick Mylar central layer designed to simulate the presence of a lateral gauge package. The recession velocity was found to vary significantly further from the central encapsulation layer, implying that the presence of this layer had modified the induced shock. Further, it 
was found that the same code employed by Winter and Harris $^{23}$ allowed similar recession velocities to be predicted.

Overall, while there is an established body of work $^{6,15,18,25}$ that interprets lateral gauge results according to the approach explained here, it is clear from the work of Winter and Harris ${ }^{23}$ and Winter et al. ${ }^{24}$ that some doubt as to the validity of this approach remains. It is also important to point out that, while their results have been established over a number of years, much of the work on lateral gauges ${ }^{6,15,18,25}$ comes from a selected group within the shock physics community who have investigated this area. It is only recently that this fundamental work has encouraged wider elements of the community to begin to turn their attention to this important field leading to possible alternative explanations for the observed lateral gauge response. Therefore care should be taken in lateral gauge interpretationparticularly in relation to inference of any strengthening effects.

\section{CONCLUSIONS}

The shock response of G13 WC-Co has been studied using plate impact experiments at impact velocities in the range $199-857 \mathrm{~m} / \mathrm{s}$. Both longitudinal and lateral gauge shots were undertaken and comparison made to previous data for similar grades of cemented WC. G13 WC-Co has been found to exhibit a $U_{s}-U_{p}$ relationship with an initial elastic region extending beyond the particle velocity associated with the HEL of WC-Co followed by a negative gradient in the region $0.03 \mathrm{~mm} / \mu \mathrm{s} \leq U_{p} \leq 0.36 \mathrm{~mm} / \mu \mathrm{s}$. It was suggested that the initial elastic regime likely extends above that of WC-Co due to the composite nature of $\mathrm{WC}-\mathrm{Co}$, with elastic waves continuing to propagate through the WC phase. The negative ramped region was partially attributed to detection of the elastic precursor, which had a higher velocity in this regime. However, the continued negative gradient of the Hugoniot after the HEL values of both G13 WC-Co and pure WC were detected meant that another explanation was required. Given that the elastic limit appeared to range from that of WC-Co to that of the WC phase only, it was proposed that a mechanism such as deformation hardening of the WC particles, which reached saturation at $U_{p}=0.37 \mathrm{~mm} / \mu \mathrm{s}$ where the principal Hugoniot was rejoined, might be a reasonable alternative explanation. A three-stage profile was observed in longitudinal stress gauge traces consisting of an initial elastic rise to the HEL of G13 WC-Co followed by a rise to a point of inflection, which was shown to correspond to the HEL of pure WC, and finally a plastic ramp up to a maximum stress. The lack of a final plastic rise exhibited for similar WC-Co cermets in the literature was attributed to the fact that impact stresses were not high enough to cause the shock to overtake the elastic precursor. This was confirmed by the experimentally derived $U_{s}-U_{p}$ relationship, which clearly showed that all experimental datapoints lie on either the initial elastic or intermediate plastic regimes. The plastic ramped behavior was tentatively linked to deformation hardening as the Co matrix was compressed. An average G13 WC-Co HEL of $3.3 \pm 0.2 \mathrm{GPa}$ was calculated, backed by estimates from the measured shear strength. This was slightly lower than reported elsewhere for WC-5Co, a factor attributed to the relatively high Co content of G13 WC-Co. Spall strength was found to be $4.38 \mathrm{GPa}$, around $50 \%$ higher than in WC-5Co grades (again, it was suggested that this was linked to the Co content-it is postulated that a higher Co content led to a less brittle structure).

Lateral gauge results have, to the authors' knowledge, not been previously reported for WC-Co and showed a number of interesting features. A ramp during the initial rise of the shock and an apparent weakening phenomenon over the duration of the shock were both observed. While recent work investigating the lateral gauge approach has suggested that the latter effect may be an artifact of sample preparation, a large body of lateral gauge results for other materials (albeit largely produced by a similar set of workers who have previously worked to ignite interest in this field) combined with the strong nature of $\mathrm{WC}-\mathrm{Co}$ suggests that this increase in lateral stress/decrease in shear strength arises due to plastic deformation behind the shock. The initial ramp may be attributed to the arrival of an elastic precursor or possibly to pressure hardening effects. It is also possible that the relatively lazy rise times exhibited by the lateral gauge traces are a feature of the composite nature of $\mathrm{WC}-\mathrm{Co}$, with the shock wave a superposition of those in the WC itself and the Co matrix. Lateral stresses have been used to establish the stress dependence of the shear strength of G13 WC-Co, which is observed to vary approximately linearly with impact stress. This increase continues beyond the HEL, but with a lower gradient, providing evidence for both maintenance of strength and confirming the occurrence of plastic deformation.

\section{ACKNOWLEDGMENTS}

This research has been funded by the Dutch Ministry of Defense, partly through the Research Program "V518Munitions and Weapons Effects."

${ }^{1}$ E. Lassner and W.-D. Schubert, Tungsten: Properties, Chemistry, Technology of the Element, Alloys, and Chemical Compounds (Kluwer Academic/ Plenum, New York, 1999).

${ }^{2} \mathrm{~V}$. John, Introduction to Engineering Materials (Palgrave Macmillan, Hampshire, UK, 2003).

${ }^{3}$ P. J. Hazell, S. E. Donoghue, C. J. Roberson, and P. L. Gotts, Advances in Ceramic Armor: A Collection of Papers Presented at the 29th International Conference on Advanced Ceramics and Composites, Ceramic Engineering and Science Proceedings, Cocoa Beach, FL, 23-28 January, edited by J. J. Swab, D. Zhu, and W. M. Kriven (Wiley, New York, 2006), Vol. 26, pp. 143-150.

${ }^{4}$ C. J. Roberson, P. J. Hazell, P. L. Gotts, I. M. Pickup, and R. Morrell, Advances in Ceramic Armor: A Collection of Papers Presented at the 29th International Conference on Advanced Ceramics and Composites, Ceramic Engineering and Science Proceedings, Cocoa Beach, FL, 23-28 January, edited by J. J. Swab, D. Zhu, and W. M. Kriven (Wiley, New York, 2006), Vol. 26, pp. 151-159.

${ }^{5}$ D. P. Dandekar and D. E. Grady, in Shock Compression of Condensed Matter, edited by M. D. Furnish, N. N. Thadhani, and Y. Horie (AIP, New York, 2001), pp. 783-786.

${ }^{6}$ J. C. F. Millett, N. K. Bourne, and D. P. Dandekar, J. Appl. Phys. 96, 3727 (2004).

${ }^{7}$ D. Grady, Int. J. Impact Eng. 23, 307 (1999).

${ }^{8}$ S. P. Marsh, LASL Shock Hugoniot Data (University of California Press, Los Angeles, CA, 1980).

${ }^{9}$ T. J. Holmquist, A. M. Rajendran, D. W. Templeton, and K. D. Bishnol, "A Ceramic Armor Material Database," TARDEC Technical Report No. 
13754, 1999.

${ }^{10}$ D. P. Dandekar, "Spall Strength of Tungsten Carbide," Report No. ARLTR-3335, 2004.

${ }^{11}$ K. J. Frutschy and R. J. Clifton, Exp. Mech. 38, 116 (1998).

${ }^{12}$ N. K. Bourne, Mater. Sci. Technol. 14, 273 (2003).

${ }^{13}$ Z. Rosenberg, D. Yaziv, and Y. Partom, J. Appl. Phys. 51, 3702 (1980).

${ }^{14}$ M. A. Meyers, Dynamic Behaviour of Materials (Wiley, New York, 1994).

${ }^{15}$ G. T. Gray, N. K. Bourne, and J. C. F. Millett, J. Appl. Phys. 94, 6430 (2003).

${ }^{16}$ Z. Rosenberg and Y. Partom, J. Appl. Phys. 58, 3072 (1985).

${ }^{17}$ Z. Rosenberg and N. S. Brar, J. Appl. Phys. 77, 1143 (1995).

${ }^{18}$ J. C. F. Millett, N. K. Bourne, and Z. Rosenberg, J. Phys. D 29, 2466
(1996).

${ }^{19}$ P. J. Hazell, C. Stennett, and G. Cooper, Polym. Compos. 29, 1106 (2008).

${ }^{20}$ B. Klein, N. Frage, M. P. Dariel, and E. Zaretsky, J. Appl. Phys. 93, 968 (2003).

${ }^{21}$ Y. J. E. Meziere, J. C. F. Millett and N. K. Bourne, J. Appl. Phys. 100, 033513 (2006)

${ }^{22}$ Z. Zhang, K. Fukuoka, M. Kikuchi, M. Kodama, K. Shibata, and T. Mashimo, Int. J. Impact Eng. 32, 643 (2005).

${ }^{23}$ R. E. Winter and E. J. Harris, J. Phys. D 41, 035503 (2008).

${ }^{24}$ R. E. Winter, G. D. Owen, and E. J. Harris, J. Phys. D 41, 202006 (2008).

${ }^{25}$ J. C. F. Millett, N. K. Bourne, and N. R. Barnes, J. Appl. Phys. 92, 6590 (2002). 